\title{
Two-Stage Optimal Scheduling of Electric Vehicle Charging based on Transactive
} Control

\author{
Liu, Zhaoxi; Wu, Qiuwei; Ma, Kang; Shahidehpour, Mohammad ; Xue, Yusheng; Huang, Shaojun
}

Published in:

IEEE Transactions on Smart Grid

Link to article, DOI:

10.1109/TSG.2018.2815593

Publication date:

2018

Document Version

Peer reviewed version

Link back to DTU Orbit

Citation (APA):

Liu, Z., Wu, Q., Ma, K., Shahidehpour, M., Xue, Y., \& Huang, S. (2018). Two-Stage Optimal Scheduling of Electric Vehicle Charging based on Transactive Control. IEEE Transactions on Smart Grid, 10(3), 2948 - 2958. https://doi.org/10.1109/TSG.2018.2815593

\section{General rights}

Copyright and moral rights for the publications made accessible in the public portal are retained by the authors and/or other copyright owners and it is a condition of accessing publications that users recognise and abide by the legal requirements associated with these rights.

- Users may download and print one copy of any publication from the public portal for the purpose of private study or research.

- You may not further distribute the material or use it for any profit-making activity or commercial gain

- You may freely distribute the URL identifying the publication in the public portal 


\title{
Two-Stage Optimal Scheduling of Electric Vehicle Charging based on Transactive Control
}

\author{
Zhaoxi Liu, Member, IEEE, Qiuwei Wu, Senior Member, IEEE, Kang Ma, Member, IEEE, \\ Mohammad Shahidehpour, Fellow, IEEE, Yusheng Xue, Member, IEEE, and Shaojun Huang, Member, IEEE
}

\begin{abstract}
In this paper, a two-stage optimal charging scheme based on transactive control is proposed for the aggregator to manage day-ahead electricity procurement and real-time EV charging management in order to minimize its total operating cost. The day-ahead electricity procurement considers both the day-ahead energy cost and expected real-time operation cost. In the real-time charging management, the cost of employing the charging flexibility from the EV owners is explicitly modelled. The aggregator uses a transactive market to manage the real-time charging demand to provide the regulating power. A model predictive control (MPC) based method is proposed for the aggregator to clear the transactive market. The realtime charging decisions of the EVs are determined by the clearing of the proposed transactive market according to the realtime requests and preferences of the EV owners. As such, the aggregators decisions in the real-time EV charging management and regulating power markets can be optimized. At the same time, the charging requirements and response preferences of the EV owners are respected. Case studies using real world driving data from the Danish National Travel Surveys were conducted to verify the proposed framework.
\end{abstract}

Index Terms-Electric vehicles (EVs), regulating power, transactive control, transactive energy, two-stage optimization.

\section{INTRODUCTION}

$\mathbf{T}$ HE electric vehicle (EV) markets of many countries have been growing rapidly in recent years [1]. A series of national plans and regional projects have been put forward to further promote the EV deployment [2], [3]. The EV is considered as a promising alternative to conventional internal combustion engine (ICE) vehicles around the world because it can not only reduce the green house gas (GHG) emission from the transportation sector but also utilize excessive electric power from renewable energy sources (RESs) [4].

This work was supported by the ForskEL programme through the "IDE4LDK Top-Up" project.

Z. Liu, and S. Huang are with Center for Electric Power and Energy (CEE), Department of Electrical Engineering, Technical University of Denmark, Kgs. Lyngby, 2800 Denmark (e-mail: zhliu@elektro.dtu.dk, shuang@elektro.dtu.dk).

Q. Wu is with Center for Electric Power and Energy (CEE), Department of Electrical Engineering, Technical University of Denmark, Kgs. Lyngby, 2800 Denmark (e-mail: qw@elektro.dtu.dk), and also with the Harvard China Project, School of Engineering and Applied Sciences, Harvard University, Cambridge, MA 02138, USA (qiuwu@ seas.harvard.edu).

K. Ma is with University of Bath, Bath, U.K. (e-mail: K.Ma@bath.ac.uk).

M. Shahidehpour is with Robert W. Galvin Center for Electricity Innovation, Illinois Institute of Technology, Chicago, IL 60616 USA, and also with King Abdulaziz University, Jeddah 21589, Saudi Arabia (e-mail: ms@iit.edu).

Y. Xue is with State Grid Electric Power Research Institute, Nanjing 210003, China (e-mail: xueyusheng@ sgepri.sgcc.com.cn).

Corresponding author: Q. Wu (e-mail: qw@elektro.dtu.dk).
Due to the great impact of the large-scale integration of EVs into the power system, it has attracted a lot of attention from both the industry and academia [5], [6]. Particularly, the optimal EV charging scheduling have been widely researched in order to exploit the flexibility of the charging demand [7]. A number of optimal charging strategies of the EV aggregators were studied to minimize the charging cost and maximize the integration number of the charging EVs in [8]-[13]. The EV charging was optimized to limit the peak demand in the grid and fill the valley in [14]-[17]. Further, the optimal scheduling of the EV charging was studied in [18]-[20] to provide ancillary services including frequency regulation and spinning reserves to the grid. The study in [21] scheduled the EV charging demand to improve the system reliability of the distribution network. The optimal charging schemes of the EV aggregators were studied to address the congestion problems in distribution networks in [22], [23]. In [11], [24], [25], the optimal EV charging was investigated to cope with the uncertainty from the RESs.

Valuable insights of the optimal EV charging in a variety of contexts were provided in previous studies. However, the existing optimal charging schemes are implemented with the charging control strategies directly distributed by the EV aggregator or fleet operator (FO), and the customers need to provide the aggregator or FO with detailed charging requirements including the charging energy and arrival/departure plans for the day-ahead or real-time optimization. In practice, there should be incentives for the EV owners to provide their charging flexibility. Specifically, the aggregator need to pay the EV owners for delaying the charging. Therefore, there is a cost of the aggregator to use the charging flexiblity of the EVs. In such schemes, the cost of the aggregator to employ the flexibility of the EV charging from the customers is not explicitly modelled. Moreover, each EV owner has different requirements on the charging and preferences for its response in practice. The charging requirements and response preferences of the EV owners should have the first priority and be respected by the control of the aggregator. Otherwise, the willingness of the customers to provide flexibility would decrease. Thus, an efficient EV charging framework is required by the aggregator so that the cost of employing the flexibility of the EV charging can be explicitly reflected and the EV oweners' own preferences can be respected. As such, the EV aggregator can consider the cost of employing the flexibility of the EV charging in its optimization. Meanwhile, the EV owners can get reimbursed based on their preferred rates for the responses and their charging requirements can be guaran- 
teed. In order to meet such challenges, the EV aggregator is studied in this paper. A two-stage optimal charging scheme based on transactive control is proposed for the aggregator to manage day-ahead electricity procurement and real-time EV charging management.

The transactive control concept was proposed by a number of research groups and tested in a few pilot projects for the building and residential energy management. The pilot projects have shown positive results on the coordination of the distributed demand such as heat pumps (HPs), heating, ventilation and air conditioning (HVAC) system of buildings and household appliances [26]-[30]. Transactive control is generally defined as an incentive signaling approach utilizing an economic signal as the primary basis for communicating the desire to change the operational state of responsive assets [26]. Instead of direct control orders, the decisions in the transactive control approach are made based on values. Such decisions can either be analogous to or literally economic transactions. Transactive control is regarded as a kind of promising approach for the coordination of the responsive demand in the smart grids. It can fully utilize the response potential and has a certain system reaction while maintaining market efficiency and raising no privacy issue [31].

The objective of the paper is to develop a decentralized pricing mechanism between the aggregator and EV owners to explicitly reflect the cost of using flexibility from EV owners. This problem has not been studied according to the knowledge of the authors.

In order to develop such a mechanism, the transctive control concept is adopted. A transactive market is established between the EV aggregator and EV owners. The actual charging control strategies of the EVs in real time are determined according to the clearing between the aggregator and EV owners in the proposed transactive market. The EV charging response mechanism in the proposed framework is customized to accommodate the interests of both the aggregator and EV owners.

The proposed transactive framework has a number of advantages compared to the existing EV charging models for EV aggregators. Firstly, with the proposed framework, the EV aggregator is able to consider the operation cost of employing the flexibility of the EV charging from the customers dynamically in the real-time operation. As such, the proposed model can avoid potential high cost from EV owners by abusing their charging flexibility. The aggregator is therefore able to maximize its surplus while the reimbursements to the EV owners account for their actual flexibility. Secondly, the different response preferences of the EV owners are respected by the real-time operation of the aggregator. An EV owner who is more flexible gets more reimbursements while a conservative EV owner has a faster charging and less reimbursement. The proposed framework allows the EV owners to offer the EV charging flexibility based on their preferences and get reimbursed accordingly. Thirdly, the EV owners do not have to provide the privacy information (e.g., their driving plans) to the aggregator in the proposed framework.

In summary, the proposed charging framework enables the $\mathrm{EV}$ aggregator to efficiently employ the flexibility of the EV charging and maximize its surplus in the real-time operation. Meanwhile, the willingness of the EV owners to offer their flexibility is fully respected.

The contributions of this paper are summarized as follows:

- Propose a transactive market model incorporating the cost of employing the flexibility from the EV owners for the aggregator to handle the real-time charging management of the EVs. With the proposed transactive market model, the aggregator is able to quantify the cost of employing the EV owners' charging flexibility so that it can optimize its decisions in the real-time operation. Meanwhile, the EV owners are able to decide their own charging requirements and response rates in the real-time transactive market to guarantee that their driving needs and response preferences are met.

- Propose a model predictive control (MPC) based method for the aggregator to clear the transactive market. With the clearing of the transactive market, the real-time decisions of the EV charging and volumes for the aggregator to participate in the regulating power market are determined while the charging requirements of the EV owners are respected.

- Propose a two-stage stochastic charging scheduling scheme based on transactive control to manage the EV charging for the day-ahead scheduling and real-time operation of the aggregator.

The paper is organized as follows. The two-stage control framework of the EV charging for the aggregator is introduced in Section II. Particularly, the model of the transactive market organized by the aggregator to handle the flexible EV charging demand for the real-time operation is presented. The two-stage stochastic programming model of the electricity procurement in the day-ahead market for the aggregator is described in Section III. The MPC based method for the aggregator to clear the real-time transactive market is also described in the same section. In Section IV, the case studies are presented and discussed, followed by conclusions in Section V.

\section{ENERGY MANAGEMENT FRAMEWORK FOR EV AGGREGATORS}

In order to manage the EV charging for both day-ahead scheduling and real-time operation, an energy management framework is proposed for the aggregator as shown in Fig. 1. The proposed framework includes two stage: the day-ahead stage and real-time stage. In the first stage, the aggregator conducts the day-ahead planning to determine its electricity procurement in the day-ahead market. The aggregator purchases the electricity in the day-ahead market by minimizing the electricity procurement cost and expected real-time operation cost of the EV charging. In the second stage, the aggregator organizes a transactive market with the EV owners and participates in the regulating power market when it is profitable in the real-time operation. In the transactive market, the EV owners submit the charging targets and response preferences of the current time interval. With the procured volumes in the day-ahead electricity market and prices of the regulating power market, the aggregator conducts the realtime optimization to determine the clearing price for the $\mathrm{EV}$ 
owners' responses in the transactive market and the volume to participate in the regulating power market of the current interval. With the clearing price of the transactive market, the response volumes of the EV owners in the interval are determined. The actual charging demand of the EV owners in the interval is determined by the proposed charging targets and the response volumes. The EV owners are reimbursed by the aggregator for their response volumes with the clearing price of the transactive market in the interval.

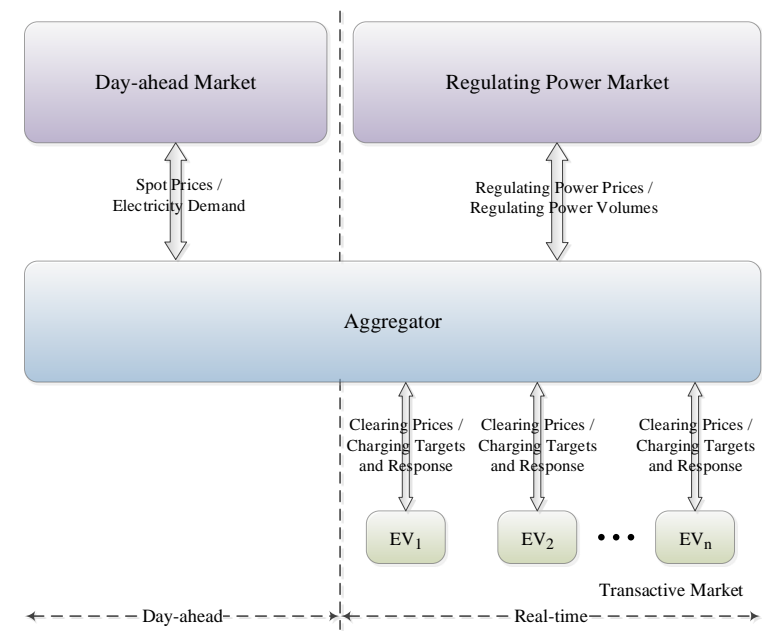

Fig. 1. System Framework Diagram of EV Charging for Aggregators.

In the transactive market, the EV owners submit their charging targets and response curves of the interval. The response curve of the EV owners is illustrated by Fig. 2.

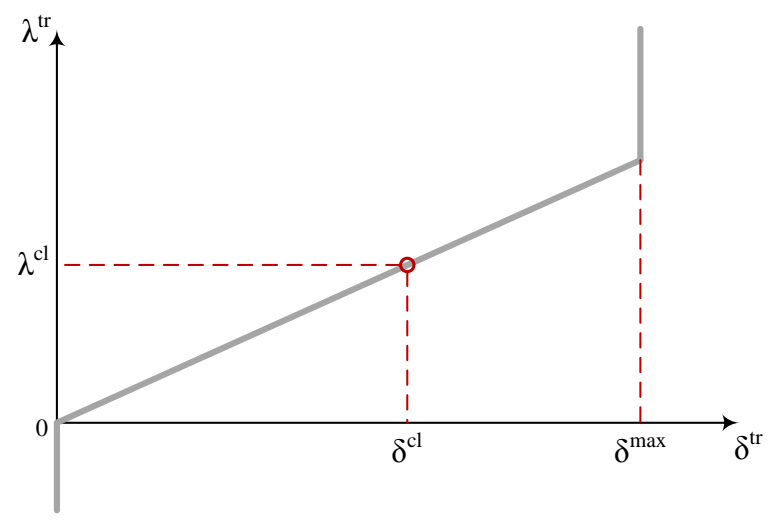

Fig. 2. Illustration of the EV Response Curves in the Transactive Market.

When there are no incentives, the EV owners tend to charge the EVs as soon as possible till they are fully charged. By joining the transactive market, the EV owners offer the aggregator the permission to reduce their charging demand of the interval according to their own response curves. Thus, the actual charging demand in the interval is the targeted charging demand of the EV owners $q_{v, t}^{t a r}$ minus the reduced demand $\delta_{v, t}^{c l}$ as (1).

$$
q_{v, t}=q_{v, t}^{t a r}-\delta_{v, t}^{c l}
$$

In every interval, the aggregator clears the market at a price $\lambda_{t}^{c l}$ according to the real-time optimization and broadcasts the price to all the EV owners. The responses of the EV owners are determined according to their response curves and the clearing price as shown in Fig. 2 and (2). The coefficient $\theta_{v, t}$ in (2) is the inverse of the response curve's slope, which is a customersetting parameter. A larger $\theta_{v, t}$ means the EV owner is more willing to reduce the demand with the same clearing price.

$$
\delta_{v, t}^{c l}=\left\{\begin{array}{cc}
\theta_{v, t} \lambda_{t}^{c l} & \left(\lambda_{t}^{c l} \leqslant \frac{\delta_{v, t}^{\max }}{\theta_{v, t}}\right) \\
\delta_{v, t}^{\max } & \left(\lambda_{t}^{c l}>\frac{\delta_{v, t}^{\max }}{\theta_{v, t}}\right)
\end{array}\right.
$$

When the clearing price of the market and reduced demand of the EV owners are determined, the cost of the EV charging for the EV in the interval is calculated as (3). It is equal to the charging electricity with the base charging price $\lambda^{b a}$ minus the reimbursement for the reduced demand with the clearing price of the transactive market in the interval.

$$
\operatorname{COST}_{v, t}=\lambda^{b a} q_{v, t}-\lambda_{t}^{c l} \delta_{v, t}^{c l}
$$

In every interval, the EV owner can adjust its preferences in the transactive market. For example, an EV owner may decrease its response rate and be more conservative to provide flexibility due to the unexpected driving activity. In this case, the EV will have a lower reduced demand $\delta_{v, t}^{c l}$ by the clearing of the real-time market as (2). As a result, the aggregator will reduce less of its charging demand and charge the EV faster in the interval to suit the needs of the EV owner as (1). Accordingly, the EV owner will receive a lower reimbursement from the aggregator according to the clearing of the transactive market because its charging demand is reduced less than the original case. Thus, the proposed model encourages the EV owner to provide as much flexibility as possible to maximize his/her economic benefits. Meanwhile, the EV owner has the option to adjust its preferences in the real-time operation to secure its driving needs.

In order to provide a more flexible scheme for the EV owners, the response model can be extended to a piecewise linear one. The piecewise linear response curve of the EV owners is illustrated in Fig. 3.

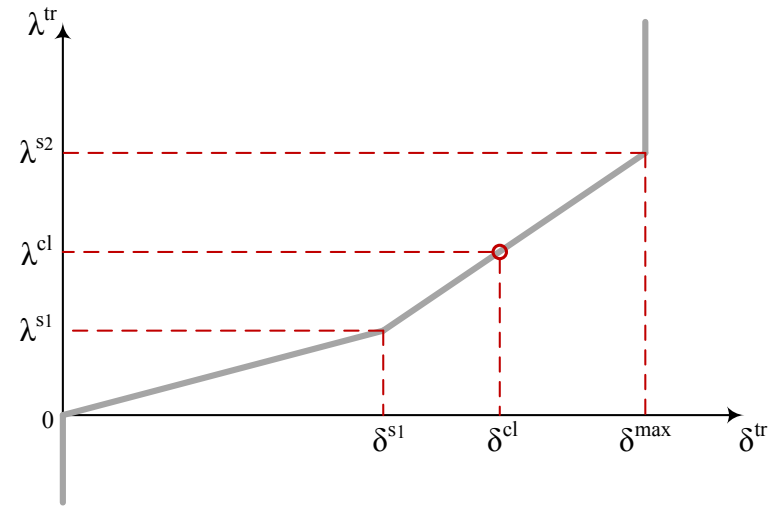

Fig. 3. Illustration of the Piecewise-linear EV Response Curves in the Transactive Market. 
In this case, the responses of the EV owners are determined according to (4).

$$
\delta_{v, t}^{c l}=\left\{\begin{array}{cc}
\theta_{v, t}^{s_{1}} \lambda_{t}^{c l} & \left(\lambda_{t}^{c l} \leqslant \lambda_{v, t}^{s_{1}}\right) \\
\delta_{v, t}^{s_{1}}+\theta_{v, t}^{s_{2}}\left(\lambda_{t}^{c l}-\lambda_{v, t}^{s_{1}}\right) & \left(\lambda_{v, t}^{s_{1}}<\lambda_{t}^{c l} \leqslant \lambda_{v, t}^{s_{2}}\right) \\
\delta_{v, t}^{\text {max }} & \left(\lambda_{t}^{c l}>\lambda_{v, t}^{s_{2}}\right)
\end{array}\right.
$$

where $\theta_{v, t}^{s_{1}}$ and $\theta_{v, t}^{s_{2}}$ in (4) are the inverse of the response curve's slopes set by the EV owners.

In order to provide the EV flexibility to the grid, the aggregator joins the regulating power market in the realtime operation when it is profitable. The proposed framework can be integrated into the real-time regulating power market, e.g., the real-time market framework proposed in Ecogrid EU project [32]. The real-time regulating power market operator calculates and broadcasts the real-time regulating price and price forecast of the following few intervals to call for the necessary upward/downward regulating power according to the needs of the system operator to handle the issues such as the imbalance and congestion. The market participant, which is the EV aggregator in this paper, responds to the prices and provides the upward/downward regulating power to the grid according to its own interest.

\section{Problem Formulation of the Two-Stage SCHEDUling Model For EV AgGREgators}

\section{A. Day-ahead Planning of EV Aggregator}

The day-ahead planning is conducted by the aggregator to determine its electricity procurement in the day-ahead market. For the aggregator, the day-ahead planning aims to maximize the aggregator's own profit considering the possible cost of utilizing the flexibility of the EV charging demand in the real-time transactive market. In the study, the EV aggregator is assumed to be a price-taker in the pool-based day-ahead market. The aggregator carries out its day-ahead planning with the forecast electricity prices of the market. The optimization problem of the aggregator can be formulated as a two-stage stochastic model as follows.

$$
\min \boldsymbol{\lambda}_{\mathbf{d a}}^{T} \mathbf{x}+E_{\omega}[\mathrm{Q}(\mathbf{x}, \boldsymbol{\omega})]
$$

Subject to

$$
\sum_{t \in \mathcal{T}} x_{t}=E_{\omega}\left[\sum_{t \in \mathcal{T}} \sum_{v \in \mathcal{V}} \mu_{v} d_{v, t, \omega}\right]
$$

where $\boldsymbol{\lambda}_{\mathrm{da}}$ is the vector of the electricity prices in the dayahead market; $\mathbf{x}=\left\{x_{t}: \forall t \in \mathcal{T}\right\}$ is the vector of the procured electricity by the aggregator in the day-ahead market; $\omega$ is the realization with respect to the EV driving pattern probability space $(\Omega, P) ; d_{v, t, \omega}$ denotes the driving distance of the EVs in scenario $\omega ; \mu_{v}$ is the EV electricity consumption rate. The objective of the aggregator's day-ahead planning is to minimize its cost subjected to the daily energy balance constraint (6), with which the total energy procured in the day-ahead market is equal to the expected total driving energy consumption of the EVs in the following day. Specifically, the first term in the objective (5) is the cost of procuring electricity in the day-ahead market by the aggregator. The second term is the expected cost of the aggregator in the real-time operation, and the function $Q(\mathbf{x}, \boldsymbol{\omega})$ can be expressed as follows.

$$
\begin{aligned}
& Q(\mathbf{x}, \boldsymbol{\omega})= \\
& \min \boldsymbol{\lambda}_{\mathbf{c l}}^{T} \sum_{v \in \mathcal{V}} \boldsymbol{\delta}_{v}-\lambda^{\mathrm{ba}} \mathbf{1}^{T} \sum_{v \in \mathcal{V}} \mathbf{q}_{v}+\boldsymbol{\lambda}_{\mathbf{r p}}^{T}\left(\sum_{v \in \mathcal{V}} \mathbf{q}_{v}-\mathbf{x}\right)
\end{aligned}
$$

Subject to

$$
\begin{gathered}
\mathbf{s o c}_{v}^{\text {min }} \leqslant \mathbf{s o c}_{v, \omega} \leqslant \mathbf{s o c}_{v}^{\max } \quad \forall v \in \mathcal{V} \\
\operatorname{soc}_{v, t, \omega}=\left[q_{v, t} s_{v, t, \omega}-\mu_{v} d_{v, t, \omega}\left(1-s_{v, t, \omega}\right)\right] / B_{v} \\
+s o c_{v, t-1, \omega} \quad \forall t \in \mathcal{T} \quad \forall v \in \mathcal{V} \\
\mathbf{q}_{v} \leqslant p_{v}^{\max } \mathbf{s}_{v, \omega} \Delta t \quad \forall v \in \mathcal{V} \\
\mathbf{q}_{v} \geqslant \mathbf{0} \quad \forall v \in \mathcal{V}
\end{gathered}
$$

where $\lambda_{\mathrm{cl}}$ is the vector of the clearing prices in the realtime transactive market organized by the aggregator; $\boldsymbol{\delta}_{v}=$ $\left\{\delta_{v, t}: \forall t \in \mathcal{T}\right\}$ is the vector of the reduced demand of EV $v$ according to the clearing of the transactive market; $\mathbf{q}_{v}=\left\{q_{v, t}: \forall t \in \mathcal{T}\right\}$ is the vector of the charging electricity of EV $v ; \lambda_{\mathbf{r p}}$ is the vector of the regulating prices in the realtime regulating power market; $\operatorname{soc}_{v, \omega}=\left\{\operatorname{soc}_{v, t, \omega}: \forall t \in \mathcal{T}\right\}$ is the vector of the state-of-charge (SOC) levels of EV $v$ in scenario $\omega ; \mathbf{s}_{v, \omega}=\left\{s_{v, t, \omega}: \forall t \in \mathcal{T}\right\}$ is the vector of the EV charging availability indicators, $s_{v, t, \omega}$ shows the status of EV $v$ at time $t$ in scenario $\omega$. It is equal to 1 when the $\mathrm{EV}$ is parked and available for charging, and is equal to 0 when the $\mathrm{EV}$ is not available for charging, e.g., when it is being driven on the road; $B_{v}$ is the EV battery capacity.

The second stage objective (7) minimizes the expected cost of the aggregator in the real-time operation subject to the charging requirements of the EVs. The first term in the objective (7) is the cost of the aggregator to reduce the EV charging demand of the EV owners in the transactive market. The second term is the EV charging revenue of aggregator from the EV owners. The third term is the balance of the aggregator to participate in the real-time regulating power market. For the SOC limit constraint (8), the SOC levels of the $\mathrm{EV}$ batteries are within the specified range with the charging plan. In each time interval, the SOC levels of the batteries are calculated through the charging energy and driving energy consumption of the EVs as (9). For the charging energy limit constraint (10), the EV charging energy is constrained by the maximum power limit and the EV charging availability. The charging energy flow is constrained unidirectional by (11) as the V2G technology is not considered in the study.

The clearing prices $\boldsymbol{\lambda}_{\mathbf{c l}}$ and reduced demand $\boldsymbol{\delta}_{v}$ in the first term of the second stage objective (7) are determined in the real-time transactive market organized by the aggregator according to the response preferences of the EV owners as Fig. 2 and (2). The individual EV owners' real-time response preferences are not available to the aggregator in the dayahead planning optimization. Nevertheless, the aggregator is considered to be able to estimate the cumulative response rates of the EVs in the planning horizon when the number of EVs is considerable. The relation of the cumulative reduced 
demand and the clearing price in the transactive market can be modelled with a linear function as (12). $\boldsymbol{\Theta}_{\omega}$ in (12) is a diagonal matrix with positive elements, which are the cumulative response rates of all the $\mathrm{EV}$ owners while $\boldsymbol{\zeta}_{\omega}$ is the vector of the residuals. The elements of $\boldsymbol{\Theta}_{\omega}$ and $\zeta_{\omega}$ are learned by aggregator with the historical data.

$$
\sum_{v \in \mathcal{V}} \boldsymbol{\delta}_{v}=\boldsymbol{\Theta}_{\omega} \boldsymbol{\lambda}_{\mathbf{c l}}+\boldsymbol{\zeta}_{\omega}
$$

In practice, it is difficult for the aggregator to accurately forecast the real-time regulating prices during the planning one day ahead. The precise prediction of the regulating prices $\lambda_{\mathbf{r p}}$ in the real-time regulating power market are assumed not available to the aggregator in the day-ahead planning optimization. When the system is operated neutrally, the regulating prices have a symmetric distribution around the day-ahead spot prices [33], [34]. Therefore, the expected electricity prices in the real-time regulating power market equal the day-ahead spot prices in the same time interval. However, the distribution may have a high variance depending on the operation status of the system. Using the spot prices in the day-ahead market directly as the regulating prices in the optimization would minimize the expectation of the aggregator's objective but also increase the risk of a high cost for the aggregator in the real-time operation. In order to limit the risk of the aggregator in the real-time regulating power market, a penalty term is added in the second stage objective as (13) to constrain the possible deviation of the real-time charging consumption from the day-ahead energy plan.

$$
\begin{aligned}
& Q(\mathbf{x}, \boldsymbol{\omega})=\min \left(\boldsymbol{\lambda}_{\mathbf{c l}}^{T} \boldsymbol{\Theta}_{\omega} \boldsymbol{\lambda}_{\mathbf{c l}}+\boldsymbol{\lambda}_{\mathbf{c l}}^{T} \boldsymbol{\zeta}_{\omega}\right)-\lambda^{\mathrm{ba}} \mathbf{1}^{T} \sum_{v \in \mathcal{V}} \mathbf{q}_{v} \\
& +\boldsymbol{\lambda}_{\mathbf{d a}}^{T}\left(\sum_{v \in \mathcal{V}} \mathbf{q}_{v}-\mathbf{x}\right)+\eta\left\|\sum_{v \in \mathcal{V}} \mathbf{q}_{v}-\mathbf{x}\right\|_{2}^{2}
\end{aligned}
$$

Therefore, the optimization for the day-ahead energy planning of the aggregator can be formulated and solved using the following quadratic programming model.

$$
\begin{array}{r}
\min \boldsymbol{\lambda}_{\mathbf{d a}}^{T} \mathbf{x}+\sum_{\omega \in \Omega} \pi_{\omega}\left[\left(\boldsymbol{\lambda}_{\mathbf{c l}, \omega}^{T} \boldsymbol{\Theta}_{\omega} \boldsymbol{\lambda}_{\mathbf{c l}, \omega}+\boldsymbol{\lambda}_{\mathbf{c l}, \omega}^{T} \boldsymbol{\zeta}_{\omega}\right)\right. \\
-\lambda^{b a} \mathbf{1}^{T} \sum_{v \in \mathcal{V}} \mathbf{q}_{v, \omega}+\boldsymbol{\lambda}_{\mathbf{d a}}^{T}\left(\sum_{v \in \mathcal{V}} \mathbf{q}_{v, \omega}-\mathbf{x}\right) \\
\left.+\eta\left\|\sum_{v \in \mathcal{V}} \mathbf{q}_{v, \omega}-\mathbf{x}\right\|_{2}^{2}\right]
\end{array}
$$

Subject to

$$
\begin{gathered}
\sum_{t \in \mathcal{T}} x_{t}=\sum_{\omega \in \Omega} \pi_{\omega}\left[\sum_{t \in \mathcal{T}} \sum_{v \in \mathcal{V}} d_{v, t, \omega}\right] \\
\operatorname{soc}_{v}^{\text {min }} \leqslant \operatorname{soc}_{v, \omega}^{d a} \leqslant \mathbf{s o c}_{v}^{\text {max }} \quad \forall v \in \mathcal{V} \quad \forall \omega \in \Omega \\
\operatorname{soc}_{v, t, \omega}^{d a}=\left[q_{v, t, \omega} s_{v, t, \omega}-\mu_{v} d_{v, t, \omega}\left(1-s_{v, t, \omega}\right)\right] / B_{v} \\
+\operatorname{soc}_{v, t-1, \omega}^{d a} \quad \forall t \in \mathcal{T} \quad \forall v \in \mathcal{V} \quad \forall \omega \in \Omega
\end{gathered}
$$

$$
\begin{array}{r}
\mathbf{q}_{v, \omega} \leqslant p_{v}^{\max } \mathbf{s}_{v, \omega} \Delta t \quad \forall v \in \mathcal{V} \quad \forall \omega \in \Omega \\
\mathbf{q}_{v, \omega} \geqslant \mathbf{0} \quad \forall v \in \mathcal{V} \quad \forall \omega \in \Omega \\
\sum_{v \in \mathcal{V}} \operatorname{diag}\left[B_{v}\left(\mathbf{s o c}_{v}^{\text {max }}-\mathbf{s o c}_{v, \omega}^{d a}\right) \mathbf{s}_{v, \omega}^{T}\right] \\
=\boldsymbol{\Theta}_{\omega} \boldsymbol{\lambda}_{\mathbf{c l}, \omega}+\boldsymbol{\zeta}_{\omega} \quad \forall \omega \in \Omega
\end{array}
$$

As the solution of the quadratic programming problem above, the minimal of the model $\mathbf{x}$ gives the day-ahead planning of the aggregator by minimizing its total cost in the day-ahead market and the expected cost of the real-time operation minus the charging revenue from the EV owners. The total reduced demand of the EV owners are restricted to the gaps between the maximum energy levels and the actual energy levels of the EVs which are available for charging in the interval as (20). The EV aggregator, which can be the operator of a charging station or a parking lot with charging infrastructures or a cluster of charging piles in a residential area, is viewed as a single node to the external grid. In the case when the distribution system congestion occurs, the distribution system operator (DSO) may use the pricing signals, e.g., dynamic tariffs or distribution locational marginal prices to alleviate the congestion [22], [35], [36]. The pricing signals can be directly applied in the day-ahead prices $\boldsymbol{\lambda}_{\mathbf{d a}}$ or regulating prices $\lambda_{\mathbf{r p}}$ in the aggregator's optimization. Thus, they are not explicitly expressed in the formulation.

\section{B. Real-time EV Charging Operation of EV Aggregator}

For each interval in the real-time operation, the aggregator organizes a transactive market with the EV owners as described in Section II. The EV owners send their own charging targets and response curves to the aggregator. The aggregator clears the market according to the prices in the regulating power market and response preferences of the EV owners. The aggregator announces the clearing price of the transactive market to the EV owners. Meanwhile, the charging demand and costs of the EV owners in the interval are determined according to the clearing price as (1) and (3).

During the real-time operation, the full information is available to the aggregator which includes the charging targets and response curves of the EV owners, as well as the regulating prices in the real-time regulating power market of the current interval and the regulating price forecast of the following few intervals. With the available information, the aggregator clears the transactive market with the EV owners in order to determine the real-time control decisions of the EV charging. A MPC based approach is proposed for the aggregator to clear the transactive market in the real-time operation. As one of the most widely accepted modern control strategies, MPC naturally deals with the disturbances and uncertainties of the system [37]. In each time interval, the MPC based approach minimizes the aggregator's cost of the current interval $t$ and the expected costs of the following intervals in the prediction horizon $\mathcal{H}=\{t+1, t+2, \cdots t+H\}$ as (21). Only the charging strategy of the current interval will be implemented by the aggregator to clear the transactive market with EV owners. The solutions for the following intervals will be kept on hold. In the following interval, the aggregator updates the available 
information, conducts the optimization with the MPC based approach again and implements the decisions of the interval. The aggregator keeps performing the process to update the real-time decisions for the EV charging along the time.

$$
\min f\left(q_{v, t}\right)+\widetilde{f}\left(\widetilde{\mathbf{q}}_{v, \omega}\right)
$$

The cost of the current interval $f\left(q_{v, t}\right)$ is expressed as (22). The first term is the cost to reduce the EV owners' demand according to the clearing of the transactive market, the second term is the charging revenue from the EV owners, and the third term is the balance of the aggregator to participate in the regulating power market.

$$
\begin{aligned}
& f\left(q_{v, t}\right)= \\
& \lambda_{t}^{c l} \sum_{v \in \mathcal{V}} \delta_{v, t}^{c l}-\lambda^{b a} \sum_{v \in \mathcal{V}} q_{v, t}+\lambda_{t}^{r p}\left(\sum_{v \in \mathcal{V}} q_{v, t}-x_{t}\right)
\end{aligned}
$$

Meanwhile, $\widetilde{f}\left(\widetilde{\mathbf{q}}_{v, \omega}\right)$ is expressed as (23). It is the total expected operating cost of the aggregator in the prediction horizon from interval $t+1$ to $t+H$. The response preferences of the EV owners in the future intervals are not available to the aggregator. Thus, the empirical cumulative response of the $\mathrm{EV}$ owners is applied to estimate the cost of reducing the demand of the EV owners in the prediction horizon as the dayahead planning. $\widetilde{\mathbf{q}}_{v, \omega}$ is the vector of the estimated charging decisions of EV $v$ in the prediction horizon for scenario $\omega$, and accordingly $\widetilde{\boldsymbol{\lambda}}_{\mathbf{c l}, \omega}$ is the vector of the estimated clearing prices of the transactive market in the prediction horizon. Although $\widetilde{\mathbf{q}}_{v, \omega}$ and $\widetilde{\boldsymbol{\lambda}}_{\mathbf{c l}, \omega}$ are obtained from the solution of the optimization problem (21), it will not be implemented directly in the charging but will be updated in the optimization of the following interval when new information is available.

$$
\begin{aligned}
\tilde{f}\left(\widetilde{\mathbf{q}}_{v, \omega}\right) & =\sum_{\omega \in \Omega} \pi_{\omega}\left[\left(\widetilde{\boldsymbol{\lambda}}_{\mathbf{c l}, \omega}^{T} \boldsymbol{\Theta}_{\omega} \widetilde{\boldsymbol{\lambda}}_{\mathbf{c l}, \omega}+\widetilde{\boldsymbol{\lambda}}_{\mathbf{c l}, \omega}^{T} \boldsymbol{\zeta}_{\omega}\right)\right. \\
& \left.-\lambda^{b a} \mathbf{1}^{T} \sum_{v \in \mathcal{V}} \widetilde{\mathbf{q}}_{v, \omega}+\boldsymbol{\lambda}_{\mathbf{r p}}^{T}\left(\sum_{v \in \mathcal{V}} \widetilde{\mathbf{q}}_{v, \omega}-\mathbf{x}\right)\right]
\end{aligned}
$$

For the EV charging of the current interval, the charging strategy meets the charging requirements and response preferences of the EV owners with constraints (24)-(30). The charging requirements constraints are maintained as (24)-(27) according to the realized EV driving patterns. The response of the EV owners in the real-time transactive market is modelled by (28)-(30). A binary variable $z_{v, t}$ is introduced to indicate the status of EV $v$ in the clearing of the transactive market. When $z_{v, t}=1, \delta_{v, t}^{c l}=\delta_{v, t}^{\max }$ which means EV $v$ reaches its reduced demand upper limit according to the clearing of the transactive market. In this case, $\delta_{v, t}^{\max } \leqslant \theta_{v, t} \lambda_{t}^{c l} \leqslant \theta_{v, t} \lambda^{\max }$ by (29) where $\lambda^{\max }$ is the cap of the prices in the transactive market. When $z_{v, t}=0, \delta_{v, t}^{c l}=\theta_{v, t} \lambda_{t}^{c l}$ which means the reduced demand of EV $v$ does not reach the upper limit and it is proportional to the clearing price $\lambda_{t}^{c l}$. In this case, the clearing price $\lambda_{t}^{c l}$ is constrained as $0 \leqslant \theta_{v, t} \lambda_{t}^{c l} \leqslant \delta_{v, t}^{\max }$ by (29). The actual charging energy to be performed in the interval $q_{v, t}$ is equal to the targeted demand of the EV owners $q_{v, t}^{t a r}$ minus the reduced charging demand according to the clearing of the transactive market $\delta_{v, t}^{c l}$ as (30).

$$
\begin{aligned}
& \operatorname{soc}_{v, t}^{\min } \leqslant s o c_{v, t} \leqslant s o c_{v, t}^{\max } \quad \forall v \in \mathcal{V} \\
& \operatorname{soc}_{v, t}=\left[q_{v, t} s_{v, t}-\mu_{v} d_{v, t}\left(1-s_{v, t}\right)\right] / B_{v}+s_{o c} c_{v-1} \\
& \forall v \in \mathcal{V} \\
& q_{v, t} \leqslant p_{v}^{\max } s_{v, t} \Delta t \quad \forall v \in \mathcal{V} \\
& q_{v, t} \geqslant 0 \quad \forall v \in \mathcal{V} \\
& \delta_{v, t}^{c l}=\theta_{v, t} \lambda_{t}^{c l}\left(1-z_{v, t}\right)+\delta_{v, t}^{\max } z_{v, t} \\
& z_{v, t} \in\{0,1\} \quad \forall v \in \mathcal{V} \\
& \delta_{v, t}^{\max } z_{v, t} \leqslant \theta_{v, t} \lambda_{t}^{c l} \leqslant \delta_{v, t}^{\max }\left(1-z_{v, t}\right)+\theta_{v, t} \lambda^{\max } z_{v, t} \\
& z_{v, t} \in\{0,1\} \quad \forall v \in \mathcal{V} \\
& q_{v, t}=q_{v, t}^{t a r}-\delta_{v, t}^{c l} \quad \forall v \in \mathcal{V}
\end{aligned}
$$

When the piecewise linear response model as shown in Fig. 3 is applied, constraints (28)-(29) should be modified as follows accordingly.

$$
\begin{aligned}
& \delta_{v, t}^{c l}=\theta_{v, t}^{s_{1}} \lambda_{t}^{c l}\left(1-z_{v, t}^{s_{1}}\right)+\left[\delta_{v, t}^{s_{1}}+\theta_{v, t}^{s_{2}}\left(\lambda_{t}^{c l}-\lambda_{v, t}^{s_{1}}\right)\right] z_{v, t}^{s_{1}} \\
& +\left[\delta_{v, t}^{\max }-\delta_{v, t}^{s_{1}}+\theta_{v, t}^{s_{2}}\left(\lambda_{v, t}^{s_{1}}-\lambda_{t}^{c l}\right)\right] z_{v, t}^{s_{2}} \\
& z_{v, t}^{s_{1}}, z_{v, t}^{s_{2}} \in\{0,1\} \quad \forall v \in \mathcal{V} \\
& \lambda_{v, t}^{s_{1}} z_{v, t}^{s_{1}} \leqslant \lambda_{t}^{c l} \leqslant \lambda_{v, t}^{s_{1}}\left(1-z_{v, t}^{s_{1}}\right)+\lambda^{\max } z_{v, t}^{s_{1}} \\
& z_{v, t}^{s_{1}} \in\{0,1\} \quad \forall v \in \mathcal{V} \\
& \lambda_{v, t}^{s_{2}} z_{v, t}^{s_{2}} \leqslant \lambda_{t}^{c l} \leqslant \lambda_{v, t}^{s_{2}}\left(1-z_{v, t}^{s_{2}}\right)+\lambda^{\max } z_{v, t}^{s_{2}} \\
& z_{v, t}^{s_{2}} \in\{0,1\} \quad \forall v \in \mathcal{V}
\end{aligned}
$$

For the EV charging of the intervals in the prediction horizon $\mathcal{H}$, the estimated charging strategies meet the driving requirements and response preferences of the EV owners with constraints (31)-(35). $\widetilde{\mathbf{S O c}}_{v, \omega}=\left\{\widetilde{s O C}_{v, \tau, \omega}: \forall \tau \in \mathcal{H}\right\}$ is the vector of the estimated SOC levels of EV $v$ in the prediction horizon for scenario $\omega$. They should be within the specified range with the estimated charging decisions. Equation (36) is the equality terminal constraint for the MPC based approach. The expected average SOC level of the EVs at the end of the prediction horizon is equal to the expected average SOC level of the EVs by the day-ahead planning of the aggregator.

$$
\begin{aligned}
& \operatorname{soc}_{v}^{\text {min }} \leqslant \widetilde{\mathbf{s O c}}_{v, \omega} \leqslant \operatorname{soc}_{v}^{\max } \quad \forall v \in \mathcal{V} \quad \forall \omega \in \Omega \\
& \widetilde{s O c}_{v, \tau, \omega}=\left[\widetilde{q}_{v, \tau, \omega} s_{v, \tau, \omega}-\mu_{v} d_{v, \tau, \omega}\left(1-s_{v, \tau, \omega}\right)\right] / B_{v} \\
& +\widetilde{\operatorname{sOc}}_{v, \tau-1, \omega} \quad \forall v \in \mathcal{V} \quad \forall \tau \in \mathcal{H} \quad \forall \omega \in \Omega \\
& \widetilde{\mathbf{q}}_{v, \omega} \leqslant p_{v}^{\max } \mathbf{s}_{v, \omega} \Delta t \quad \forall v \in \mathcal{V} \quad \forall \omega \in \Omega \\
& \widetilde{\mathbf{q}}_{v, \omega} \geqslant \mathbf{0} \quad \forall v \in \mathcal{V} \quad \forall \omega \in \Omega \\
& \sum_{v \in \mathcal{V}} \operatorname{diag}\left[B_{v}\left(\mathbf{s o c}_{v}^{\max }-\widetilde{\mathbf{s o c}}_{v, \omega}\right) \mathbf{s}_{v, \omega}^{T}\right] \\
& =\boldsymbol{\Theta}_{\omega} \widetilde{\boldsymbol{\lambda}}_{\mathbf{c l}, \omega}+\boldsymbol{\zeta}_{\omega} \quad \forall \omega \in \Omega
\end{aligned}
$$




$$
\begin{gathered}
E_{\omega}\left[\sum_{v \in \mathcal{V}} B_{v}\left(s o c_{v, t+H}^{\max }-\widetilde{\operatorname{soc}}_{v, t+H, \omega}\right)\right]= \\
E_{\omega}\left[\sum_{v \in \mathcal{V}} B_{v}\left(\operatorname{soc}_{v, t+H}^{\max }-\operatorname{soc}_{v, t+H, \omega}^{d a}\right)\right]
\end{gathered}
$$

In each time interval, the new information of the driving patterns of the EVs, the response preferences of the EV owners and the prices in the regulating power market become available to the aggregator. The aggregator updates the data and solves the optimization problem (21) subject to the constraints (24)(36). Accordingly, the transactive market is cleared with the solved price $\lambda_{t}^{c l}$ between the aggregator and EV owners, and the EV charging demand of the interval $q_{v, t}$ are determined.

\section{Case Studies}

In order to demonstrate the efficacy of the proposed method, case studies were carried out. The details of the case studies are described in this section.

\section{A. Parameters in the Case Studies}

The cases of an aggregator of a parking lot with $200 \mathrm{EV}$ charging customers were performed in the case studies. The driving patterns of the EVs were obtained from the real driving data of the Danish National Travel Surveys [38]. The key parameters of the EVs are listed in Table I.

TABLE I

Key PARAMETERs OF THE EVS

\begin{tabular}{c||c}
\hline Parameter & Value \\
\hline \hline EV Battery Capacity & $30 \mathrm{kWh}$ \\
EV Charging Power Limit & $10 \mathrm{~kW}$ \\
Energy Consumption Rate & $150 \mathrm{Wh} / \mathrm{km}$ \\
Lower SOC Level Limit & $20 \%$ \\
Upper SOC Level Limit & $85 \%$ \\
\hline
\end{tabular}

The electricity prices in the day-ahead market and regulating prices in the real-time regulating power market were obtained from the historical data of the Nordic power market (NordPool) as shown in Fig. 4 [34]. The base electricity price for the EV charging is set to be $1 \mathrm{DKK} / \mathrm{kWh}$. Four types of EV owners were assumed in the case study: most conservative, less conservative, less flexible and most flexible. They were indicated as group 1-4 and assigned with different response rates in the case study. The response rate $\theta_{v}$ of the EVs in Group 1 to 4 were 400, 800, 1200 and $1600 \mathrm{kWh}^{2} / \mathrm{DKK}$, respectively. It indicates that the EV owners in Group 4 are willing to reduce more charging demand than the other three groups while Group 1 is less willing to reduce the demand in the transactive market.

Three scenarios were carried out in the case studies. Scenario 1 and 3 shows the case when the actual driving patterns of the EVs in real time fluctuate slightly around the expected driving patterns of the probability space in the aggregator's optimization. Scenario 2 shows the case when the actual EV driving patterns highly deviates from the expected driving patterns. Fig. 5 shows the mean values of the cumulative driving distance of the EVs in the case studies. The average

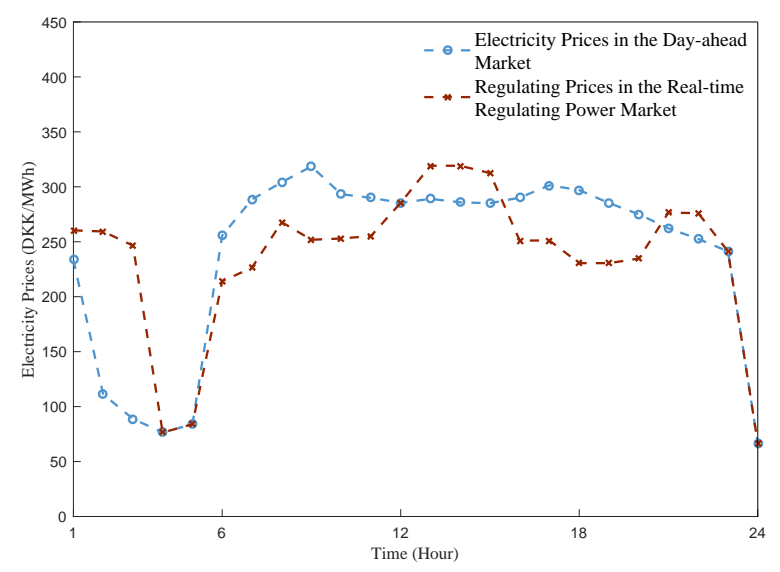

Fig. 4. Electricity Prices of the Power Market in the Case Study.

cumulative daily driving distance per EV of Scenario 1 is about $41.77 \mathrm{~km}$. This number of Scenario 2 is about $48.41 \mathrm{~km}$, which is about $15.5 \%$ higher than the expected value.

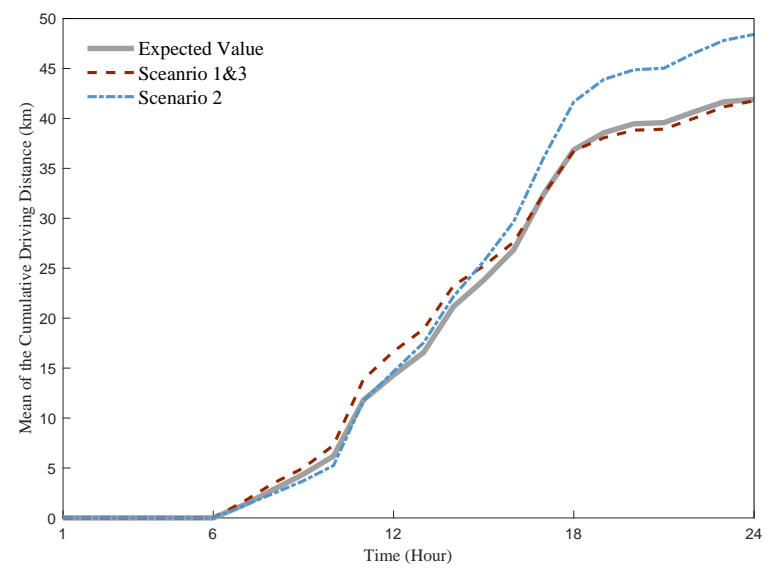

Fig. 5. Mean Value of the Cumulative Driving Distance of the EVs.

In scenario 1 and 2, the linear model for the EV response is used in the simulations. The piecewise linear model for the EV response is used in Scenario 3. In Scenario 3, the response rates $\theta_{v, t}^{s_{1}}$ in the first segment of the EV response curves are the same as the case in Scenario 1. However, the response rates $\theta_{v, t}^{s_{2}}$ in the second segment of the EV response curves are half of the values in Scenario 1, which means a higher clearing price is needed for the same amount of response from the EVs. The connection of the two segments is set at $1 \mathrm{kWh}$ in Scenario 3. It shows a case that the EV owners are more open to offer flexibility when the response demand is low. When the response demand is high, they become more conservative and ask for a higher reimbursement.

\section{B. Case Study Results}

The total demand of the day-ahead planning and real-time EV charging demand of Scenario 1 is shown in Fig. 6. As shown in the figure, the real-time charging demand at hours 2 and 3 shifts to hour 4 due to the increased regulating prices at hours 2 and 3 when the system is stressed and needs upward regulating power. With the high regulating prices in the period, 
the real-time control of the EV charging is able to reduce the charging demand and delays the charging to the period when the system is less stressed. Similarly, the real-time charging demand is reduced at hours 13-15 due to the high regulating prices in the period. On the other hand, the regulating prices are much lower than the day-ahead spot prices at hours 9, 17 to 21 . The system is with excessive generation and needs downward regulating power during the period. The realtime control increases the charging demand due to the lower regulating prices. It should also be noted that a peak demand was generated at hour 4 due to the steep regulating power prices in the first three hours. For the case of EV charging, the reduced charging demand need to be compensated in a different period so that the driving energy consumption can be met. If the day-ahead and regulating power markets result in the peak demand that causes the violations of the distribution system network constraints, extra congestion management will be implemented by the DSO to limit the peak demand. In this case, the control strategy of the aggregator would be changed. It was assumed in the case study that the network constraints of the distribution system were not violated, and the extra congestion management from the DSO is not implemented.

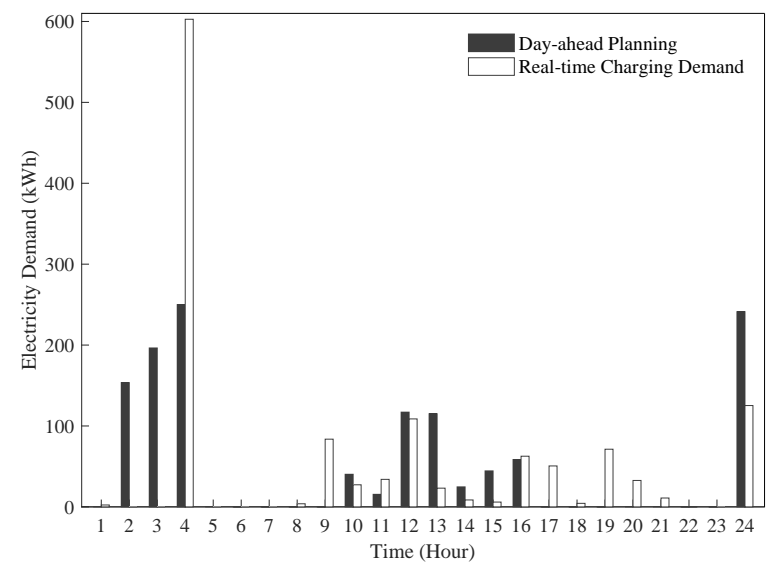

Fig. 6. EV Charging Scheduling of Scenario 1.

Besides the proposed transactive control based charging (TCBC), the uncontrolled charging (UC) and optimal charging using direct control (OCDC) of Scenario 1 were also carried out for comparison. The surplus of the aggregator with different charging schemes in Scenario 1 is shown in Table II.

TABLE II

SURPLUS OF THE AGGREGATOR IN SCENARIO 1

\begin{tabular}{c|c|c|c}
\hline (DKK) & UC & OCDC & TCBC \\
\hline \hline Payment in DA Market & -353.73 & -83.67 & -189.43 \\
Charging Revenue from Customers & 1258.08 & 1258.07 & 1258.07 \\
Reimbursement to Customers & $/$ & -150.81 & -54.75 \\
Balance in RP Market & 1.23 & 0.96 & 51.92 \\
\hline Total & 905.58 & 1024.55 & 1065.81 \\
\hline
\end{tabular}

It is shown that with the proposed transactive control based charging strategy, the aggregator has the maximum surplus. It provides a flexible strategy for the aggregator according to the response preferences of the customers available in real time.
For the uncontrolled charging, the EV owners charge the EVs without any response to the aggregator. In this case, it results in a high electricity procurement cost. For the optimal charging with direct control from the aggregator, it is assumed that the EV owners provide the aggregator with the precise forecast of the driving patterns along the day and full charging control permission of their EVs. In this case, the aggregator is able to minimize the cost of the energy procurement. However, without considering the cost to employ the flexibility of the EV charging, the reimbursement to the customers rises greatly in this case. It is worth noting that the reimbursement to customers are assumed to be determined bilaterally between the aggregator and customers with the same response rates as those in the transactive control case. However, due to the fact that the customers offers the full charging control permission of their EVs under the direct control of the aggregator, the customers shall tend to ask for a higher reimbursement for the flexibility. It will further reduce the surplus of the aggregator. When the customers' response rates are zero, the proposed transactive control based charging strategy becomes the uncontrolled charging. On the other hand, when the customers' response rates are infinite, the proposed transactive control based charging strategy has the same result as the optimal charging strategy with direct control. The proposed method offers the aggregator the optimal charging strategy between the two ends dynamically according to the response preferences of the customers.

With a higher response rate, the customer is more willing to offer the flexibility. It results in a higher reimbursement and therefore a lower charging cost. The box plot of the charging prices for the customers with the reimbursement in the real-time transactive market is shown in Fig. 7. As shown in the figure, the customers with higher response rates end up with obviously lower average charging costs. The proposed method is able to quantify the reimbursement according to the contribution of the customers to provide the flexibility. It encourages the customers to increase the response rates and offer more flexibility. It should be noted that, with a higher response rate, the customer tends to have a lower SOC level of the EV. It will impact the customer's sense of the driving endurance security. Therefore, the customers will determine their response rates according to their own confidence of the driving endurance security instead of a unified response rate of all the customers. The proposed method enables the aggregator to recognize such distinction of the customers and reimburse the customers according to their own contribution to the flexibility.

In order to illustrate the performance of the proposed approach in handling the uncertainties from the EV driving patterns, Scenario 2 was conducted with a realization of the real-time EV driving patterns highly deviating from expected EV driving patterns in the aggregator's optimization. The realtime EV charging demand of Scenario 2 is shown in Fig. 8.

Due to the longer driving distance of the EVs in Scenario 2 as shown in Fig. 5, the EV charging requirements of Scenario 2 is higher than Scenario 1. However, as shown in Fig. 8, the real-time charging demand in Scenario 2 mainly increases at hour 18 when the regulating price is low and the power system 


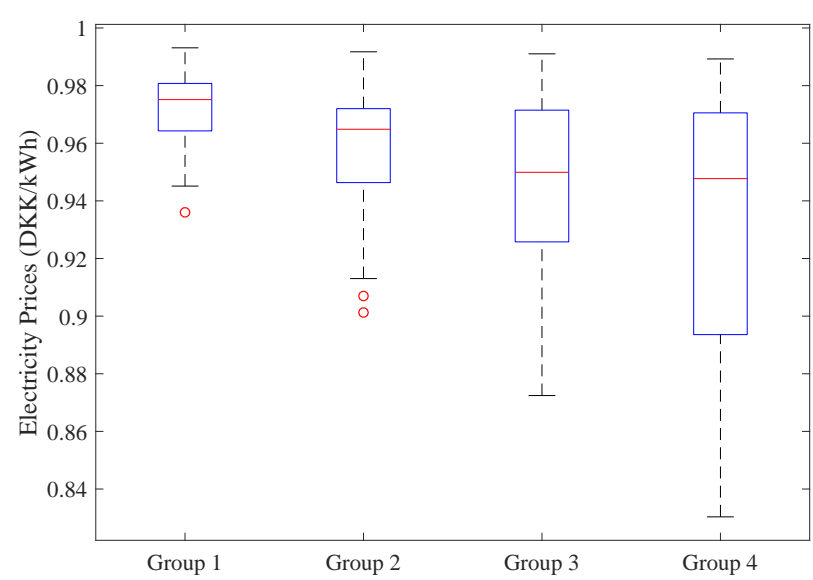

Fig. 7. Charging Prices of the Customers in Different Groups in Scenario 1.

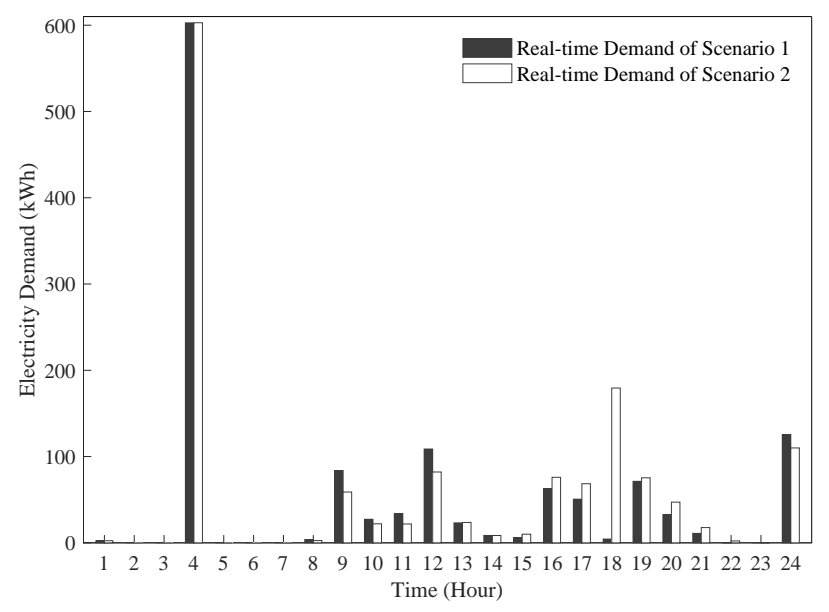

Fig. 8. Real-time EV Charging Demand of Scenario 2.

needs the downward regulation. Such behaviour of the realtime control reduces the need of the system for the downward regulating power at the moment. With the uncertainties of the EV charging requirements in real time, the proposed transactive control based approach for EV charging is able to adjust the charging in favour of the system's need while meeting the preferences of the customers. No violation of the real-time EV driving requirements occurs in Scenario 2 because the charging decisions are determined according to the clearing of the transactive market based on the customers' requests and preferences.

The results of Scenario 3 shows the case with the piecewise linear model. The real-time EV charging demand of Scenario 3 is shown in Fig. 9. As shown in the figure, the majority of the EV charging demand in Scenario 3 is very close to the case of Scenario 1. However, less charging demand is postponed in Scenario 3 which means the EVs are charged faster. It is due to the lower response rates in the second segments of the piecewise linear model in Scenario 3. Compared to Scenario 1, the $\mathrm{EV}$ owners ask for higher reimbursements when the response demand is high in this case. As a result, the aggregator tends to complete a proportion of the charging as soon as possible to limit the reimbursements to the EV owners. The solution of the aggregator respects the higher reimbursement requirements of the EV owners in the second segment of the piecewise linear model according to the clearing of the transactive market.

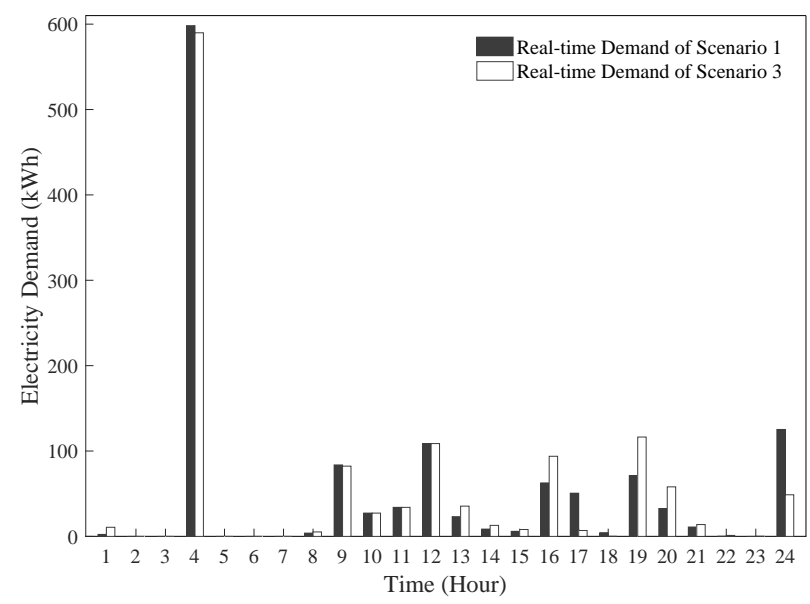

Fig. 9. Real-time EV Charging Demand of Scenario 3.

\section{CONCLusions}

In this paper, a two-stage optimal charging scheme based on transactive control is proposed for the aggregator to manage day-ahead electricity procurement and real-time EV charging management. With the advantages of the transactive control and MPC, the proposed framework for the EV aggregator is able to consider the cost of employing the flexibility from the customers quantitatively. It offers the aggregator the optimal charging solution based on the preferences of the customers dynamically in real time. It handles the uncertainties from the driving patterns of the customers without the privacy issues in the real-time charging control. It also finds the profitable options for the aggregator to participate in the regulating power market to support the grid. Meanwhile, the benefits for the customers to provide their flexibility are explicit and intuitive with the proposed approach. The reimbursement and reduced charging demand of the customers are determined by the clearing of the real-time transactive market according to their charging requirements and response preferences in every interval. Further, the customers are able to determine the degree to offer the flexibility by adjusting their own preferences in the real-time transactive market, and the outcome of the real-time control is certain for both the aggregator and customers with the clearing of the transacitve market.

In the study, the market power of the EV aggregator and uncertainty of the power market are not considered in the optimization of the aggregator. The implementation of the bidding strategies for EV aggregators [39]-[42] in the proposed transactive charging control framework will be investigated and compared in our future work. Meanwhile, the impacts of the real-time charging strategies on the EV owners' price-response settings are not considered in the study. A more sophisticated model of the real-time charging strategies' impacts on the EV owners' price-response preferences are therefore left for our future work when there is enough data from the practice or response surveys of the EV owners. 


\section{REFERENCES}

[1] P. Cazzola and M. Gorner, "Global EV Outlook 2016," International Energy Agency, Paris, France, Tech. Rep., 2016.

[2] T. Trigg and P. Telleen, "Global EV Outlook," International Energy Agency, Paris, France, Tech. Rep., 2013.

[3] Fact sheet: Plug-in electric vehicle deployment. Environmental and Energy Study Institute. [Online]. Available: http://www.eesi.org/papers/ view/fact-sheet-plug-in-electric-vehicle-deployment

[4] F. Birol, "Medium-Term Renewable Energy Market Report 2015," International Energy Agency, Paris, France, Tech. Rep., 2015.

[5] E. Valsera-Naranjo, D. Martinez-Vicente, A. Sumper, R. VillafafilaRobles, and A. Sudria-Andreu, "Deterministic and probabilistic assessment of the impact of the electrical vehicles on the power grid," in Proc. IEEE PES General Meeting, Detroit, MI, 2011, pp. 1-8.

[6] A. Dubey and S. Santoso, "Electric Vehicle Charging on Residential Distribution Systems: Impacts and Mitigations," IEEE Access, vol. 3, pp. 1871-1893, 2015.

[7] J. C. Mukherjee and A. Gupta, "A Review of Charge Scheduling of Electric Vehicles in Smart Grid," IEEE Syst. J., vol. 9, no. 4, pp. 15411553, 2015.

[8] P. You, Z. Yang, M. Y. Chow, and Y. Sun, "Optimal cooperative charging strategy for a smart charging station of electric vehicles," IEEE Trans. Power Syst., vol. 31, no. 4, pp. 2946-2956, July 2016.

[9] Y. Tang, J. Zhong, and M. Bollen, "Aggregated optimal charging and vehicle-to-grid control for electric vehicles under large electric vehicle population," IET Generation, Transmission Distribution, vol. 10, no. 8, pp. 2012-2018, 2016.

[10] L. Yang, J. Zhang, and H. V. Poor, "Risk-aware day-ahead scheduling and real-time dispatch for electric vehicle charging," IEEE Trans. Smart Grid, vol. 5, no. 2, pp. 693-702, 2014.

[11] W. Wei, F. Liu, and S. Mei, "Charging Strategies of EV Aggregator Under Renewable Generation and Congestion: A Normalized Nash Equilibrium Approach," IEEE Trans. Smart Grid, vol. 7, no. 3, pp. 1630-1641, 2016.

[12] Y. Song, Y. Zheng, and D. J. Hill, "Optimal scheduling for ev charging stations in distribution networks: A convexified model," IEEE Trans. Power Syst., vol. 32, no. 2, pp. 1574-1575, March 2017.

[13] L. Yao, W. H. Lim, and T. S. Tsai, "A real-time charging scheme for demand response in electric vehicle parking station," IEEE Trans. Smart Grid, vol. 8, no. 1, pp. 52-62, Jan 2017.

[14] B. Geng, J. K. Mills, and D. Sun, "Two-stage charging strategy for plugin electric vehicles at the residential transformer level," IEEE Trans. Smart Grid, vol. 4, no. 3, pp. 1442-1452, 2013.

[15] Z. Li, Q. Guo, H. Sun, S. Xin, and J. Wang, "A new real-time smartcharging method considering expected electric vehicle fleet connections," IEEE Trans. Power Syst., vol. 29, no. 6, pp. 3114-3115, Nov 2014.

[16] N. Chen, C. W. Tan, and T. Q. S. Quek, "Electric vehicle charging in smart grid: Optimality and valley-filling algorithms," IEEE J. Sel. Topics Signal Process., vol. 8, no. 6, pp. 1073-1083, 2014

[17] R. Wang, P. Wang, and G. Xiao, "Two-Stage Mechanism for Massive Electric Vehicle Charging Involving Renewable Energy," IEEE Trans. Veh. Technol., vol. 65, no. 6, pp. 4159-4171, 2016.

[18] J. Tan and L. Wang, "Integration of plug-in hybrid electric vehicles into residential distribution grid based on two-layer intelligent optimization," IEEE Trans. Smart Grid, vol. 5, no. 4, pp. 1774-1784, July 2014.

[19] E. Sortomme and M. A. El-Sharkawi, "Optimal Scheduling of Vehicleto-Grid Energy and Ancillary Services," IEEE Trans. Smart Grid, vol. 3 , no. 1, pp. 351-359, mar 2012.

[20] S. Han, S. Han, and K. Sezaki, "Development of an optimal vehicleto-grid aggregator for frequency regulation," IEEE Trans. Smart Grid, vol. 1, no. 1, pp. 65-72, 2010.

[21] J. Tan and L. Wang, "Enabling reliability-differentiated service in residential distribution networks with phevs: A hierarchical game approach," IEEE Trans. Smart Grid, vol. 7, no. 2, pp. 684-694, March 2016.

[22] R. Li, Q. Wu, and S. S. Oren, "Distribution Locational Marginal Pricing for Optimal Electric Vehicle Charging Management," IEEE Trans. Power Syst., vol. 29, no. 1, pp. 203-211, 2013.

[23] Z. Liu, Q. Wu, S. Oren, S. Huang, R. Li, and L. Cheng, "Distribution locational marginal pricing for optimal electric vehicle charging through chance constrained mixed-integer programming," IEEE Trans. Smart Grid, vol. PP, no. 99, pp. 1-1, 2016.

[24] Z. Li, Q. Guo, H. Sun, Y. Wang, and S. Xin, "Emission-concerned windev coordination on the transmission grid side with network constraints: Concept and case study," IEEE Trans. Smart Grid, vol. 4, no. 3, pp. 1692-1704, Sept 2013.
[25] Y. Guo, J. Xiong, S. Xu, and W. Su, "Two-Stage Economic Operation of Microgrid-Like Electric Vehicle Parking Deck," IEEE Trans. Smart Grid, vol. 7, no. 3, pp. 1703-1712, 2016.

[26] The GridWise Architecture Council, "GridWise Transactive Energy Framework," The GridWise Architecture Council, Tech. Rep. PNNL22946, 2015.

[27] S. Somasundaram, R. G. Pratt, B. A. Akyol, N. Ernandez, N. Foster, S. Katipamula, E. T. Mayhorn, A. Somani, A. Steckley, and Z. T. Taylor, "Reference Guide for a Transaction-Based Building Controls Framework," Pacific Northwest National Laboratory, Richland, WA, Tech. Rep. PNNL-23302, 2014.

[28] P. Mazza, "The Smart Energy Network: Electricity's Third Great Revolution," Climate Solutions, Olympia, WA, Tech. Rep., 2003.

[29] J. K. Kok, C. J. Warmer, and I. G. Kamphuis, "PowerMatcher: Multiagent control in the electricity infrastructure," in Proc. 4th International Joint Conference on Autonomous Agents and Multiagent Systems (AAMAS 05), Utrecht, Netherlands, 2005, pp. 75-82.

[30] K. Kok and S. Widergren, "A Society of Devices: Integrating Intelligent Distributed Resources with Transactive Energy," IEEE Power and Energy Mag., vol. 14, no. 3, pp. 34-45, 2016.

[31] Z. Liu, Q. Wu, S. Huang, and H. Zhao, "Transactive energy: A review of state of the art and implementation," in Proc. 2017 IEEE Manchester PowerTech, Manchester, UK, June 2017, pp. 1-6.

[32] Y. Ding, S. Pineda, P. Nyeng, J. Østergaard, E. M. Larsen, and Q. Wu, "Real-time market concept architecture for EcoGrid EU - A prototype for European smart grids," IEEE Trans. Smart Grid, vol. 4, no. 4, pp. 2006-2016, 2013

[33] M. Zima-Bockarjova, J. Matevosyan, M. Zima, and L. Soder, "Sharing of profit from coordinated operation planning and bidding of hydro and wind power," IEEE Trans. Power Syst., vol. 25, no. 3, pp. 1663-1673, Aug 2010.

[34] Market Data. NordPool. [Online]. Available: http://www.nordpoolspot. com

[35] S. Huang, Q. Wu, S. S. Oren, R. Li, and Z. Liu, "Distribution Locational Marginal Pricing Through Quadratic Programming for Congestion Management in Distribution Networks," IEEE Trans. Power Syst., vol. 30 , no. 4, pp. 2170-2178, 2015.

[36] L. Bai, J. Wang, C. Wang, C. Chen, and F. F. Li, "Distribution Locational Marginal Pricing (DLMP) for congestion management and voltage support," IEEE Trans. Power Syst., vol. PP, no. 99, pp. 1-1, 2017.

[37] B. Kouvaritakis and M. Cannon, Model Predictive Control, Classical, Robust and Stochastic. Springer, 2016.

[38] Q. Wu, A. H. Nielsen, J. Ostergaard, S. T. Cha, F. Marra, Y. Chen, and C. Traeholt, "Driving Pattern Analysis for Electric Vehicle (EV) Grid Integration Study," in Proc. 2010 IEEE PES Innovative Smart Grid Technologies Conf. Europe (ISGT Europe), Oct 2010, pp. 1-6.

[39] M. Zugno, J. Morales, P. Pinson, and M. Henrik, "A bilevel model for electricity retailers' participation in a demand response market environment," Energy Economics, vol. 36, pp. 182-197, March 2013.

[40] W. Wei, F. Liu, and S. Mei, "Energy pricing and dispatch for smart grid retailers under demand response and market price uncertainty," IEEE Trans. Smart Grid, vol. 6, no. 3, pp. 1364-1374, 2015.

[41] M. G. Vayá and G. Andersson, "Optimal Bidding Strategy of a Plug-In Electric Vehicle Aggregator in Day-Ahead Electricity Markets Under Uncertainty," IEEE Trans. Power Syst., vol. 30, no. 5, pp. 2375-2385, 2015.

[42] H. Wu, M. Shahidehpour, A. Alabdulwahab, and A. Abusorrah, "A game theoretic approach to risk-based optimal bidding strategies for electric vehicle aggregators in electricity markets with variable wind energy resources," IEEE Trans. Sustain. Energy, vol. 7, no. 1, pp. 374-385, 2016.

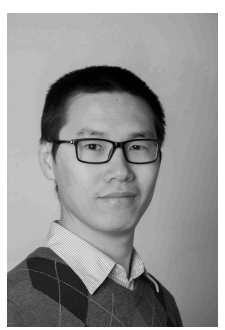

Zhaoxi Liu obtained the B.S. and M.S. degrees in electrical engineering from Tsinghua University, Beijing, China, in 2006 and 2008. He obtained the $\mathrm{Ph} . \mathrm{D}$. degree in electrical engineering from Technical University of Denmark, Denmark, in 2016.

$\mathrm{He}$ is currently a PostDoc in Center for Electric power and Energy (CEE), Department of Electrical Engineering, Technical University of Denmark. His research interests include power system operations and integration of distributed energy resources in power systems. 


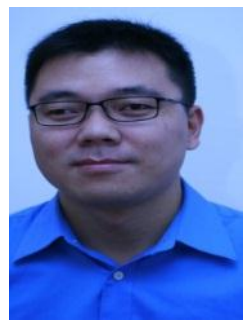

Qiuwei Wu (M'08-SM'15) obtained the B. Eng. and M. Eng. in Power System and Its Automation from Nanjing University of Science and Technology, Nanjing, China, in 2000 and 2003, respectively. $\mathrm{He}$ obtained the PhD degree in Power System Engineering from Nanyang Technological University, Singapore, in 2009.

He was a senior R\&D engineer with VESTAS Technology R\&D Singapore Pte Ltd from Mar. 2008 to Oct. 2009. He has been working at Department of Electrical Engineering, Technical University of Denmark (DTU) since Nov. 2009 (PostDoc Nov. 2009-Oct. 2010, Assistant Professor Nov. 2010-Aug. 2013, Associate Professor since Sept. 2013). He was a visiting scholar at Department of Industrial Engineering \& Operations Research (IEOR), University of California, Berkeley, from Feb. 2012 to May 2012 funded by Danish Agency for Science, Technology and Innovation (DASTI), Denmark. He was a visiting professor named by Y. Xue, an Academician of Chinese Academy of Engineering, at Shandong University, China, from Nov. 2015 to Oct. 2017. Currently, he is a visiting scholar at School of Engineering and Applied Science, Harvard University.

His research area is power system operation and control with high renewables, including wind power modelling and control, active distribution networks, and integrated energy systems. He is an Editor of IEEE Transactions on Smart Grid and IEEE Power Engineering Letters. He is also an Associate Editor of International Journal of Electrical Power and Energy Systems, Journal of Modern Power Systems and Clean Energy and IET Renewable Power Generation.

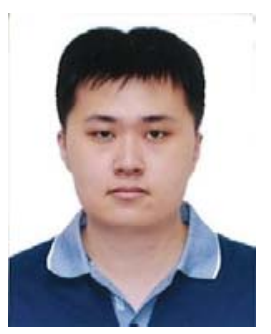

Kang Ma is now working as a lecturer at University of Bath. His research focuses on three-phase unbalanced low voltage networks. He worked as an R\&D engineer at China Electric Power Research Institute (Beijing) from 2011 to 2014, during which time he developed the first version of the reliability assessment module for a distribution network planning platform. He received his $\mathrm{PhD}$ degree in Electrical Engineering from the University of Manchester (U.K.) and his B.Eng. degree from Tsinghua University (China).

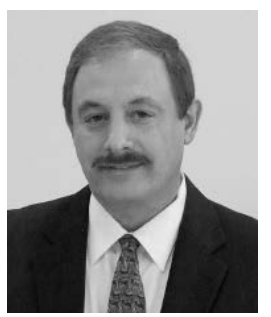

Mohammad Shahidehpour (F'01) is the Bodine Chair Professor with the Department of Electrical and Computer Engineering, the Director of the Robert W. Galvin Center for Electricity Innovation, and an Associate Director of WISER with the Illinois Institute of Technology, Chicago, IL, USA.

He was a recipient of the IEEE PES Outstanding Power Engineering Educator Award, the IEEE PES Outstanding Engineer Award, and the Chicago Chapter. $\mathrm{He}$ is the Holder of Nourbakhshian Endowed Chair Professorship, University of Kashan, Iran, and Otto Monsted Professorship, Technical University of Denmark. He is a Research Professor with King Abdulaziz University, Saudi Arabia, the Sharif University of Technology, Iran, as well as several universities in China, including Tsinghua University, Xian Jiaotong University, Nanjing University, North China Electric Power University, and Hunan University. He is an IEEE PES Distinguished Lecturer, and served as the VP of Publications for the IEEE Power and Energy Society, the Editor-in-Chief of the IEEE TRANSACTIONS ON POWER SYSTEMS, and the Founding Editor-in-Chief of the IEEE TRANSACTIONS ON SMART GRID.

Dr. Shahidehpour is a member of the U.S. National Academy of Engineering.

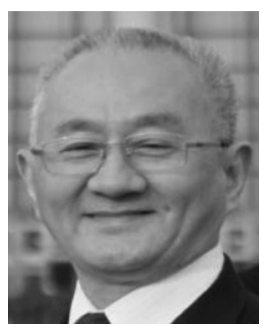

Yusheng Xue (M'88) received the M.Sc. degree in electrical engineering from EPRI, China, in 1981, and the Ph.D. degree in electrical engineering from the University of Liege, Liege, Belgium, in 1987. He was elected as an Academician of the Chinese Academy of Engineering in 1995.

$\mathrm{He}$ is now the Honorary President of State Grid Electric Power Research Institute (SGEPRI or NARI), China. He holds the positions of Adjunct Professor in many universities in China and is a Conjoint Professor with the University of Newcastle, Callaghan, NSW, Australia. He is also an Honorary Professor with the University of Queensland, Brisbane, Qld., Australia. He has been a member of the PSCC Council, and the Editorin-Chief of Automation of Electric Power System since 1999, and a Member of Editorial Board of IET Generation, Transmission, and Distribution, and Chairman of Technical Committee of Chinese National Committee of CIGRE since 2005.

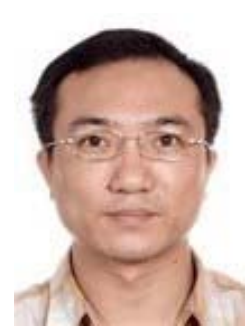

Shaojun Huang (S'13) obtained the B.Eng. from the Department of Electrical Engineering, Tsinghua University, Beijing, China, in 2001. He obtained the MSc from the Department of Energy Technology, Aalborg University, Denmark, in 2013. He obtained the PhD from the Department of Electrical Engineering, Technical University of Denmark, Denmark, in 2016.

Currently, he is a Postdoc in the Centre for Electric Power and Energy, Department of Electrical Engineering, Technical University of Denmark.

His research interests are congestion management for distribution networks with high penetration of distributed energy resources. 